\title{
Ostreobium quekettii (Codiales, Chlorophyta)
}

\author{
P. Kornmann \& P.-H. Sahling \\ Biologische Anstalt Helgoland (Meeresstation); \\ D-2192 Helgoland, Bundesrepublik Deutschland
}

\begin{abstract}
Ostreobium quekettii (Codiales, Chlorophyta). The shell-inhabiting, marine alga Ostreobium quekettii Bornet \& Flahault is a distinct species reproducing by zoospores with four flagella. Sporangium formation and the development of zoospores are described. The material obtained from Helgoland (North Sea) is assumed to be identical with the type specimen from the French Atlantic coast. $O$. quekettii is said to be of worldwide distribution; in the absence of further information, it must be doubted whether other finds reported to belong to this species are identical with the type species.
\end{abstract}

\section{EINLEITUNG}

Es gibt wohl keine Alge, über die in der Literatur so viele einander widersprechende und so wenige klare Angaben vorliegen wie von Ostreobium quekettii. Die kalkbohrende Grünalge wurde von Bornet \& Flahault (1889) in alten Austernschalen an der französischen Atlantikküste bei Le Croisic gefunden und wegen ihres röhrenförmigen Aufbaus in die Gruppe der Siphoneen eingeordnet. 1900 vertrat Nadson die Ansicht, daß die von Batters (1892) in Kalkschalen entdeckte Conchocelis rosea nur eine in der Tiefe wachsende rotgefärbte Form von Ostreobium sei. Dieser Irrtum war ein willkommenes Beispiel für die komplementäre Farbanpassung einer Grünalge an die Gedeihtiefe im Sinne der Theorie von Engelmann.

Nadsons unrichtige Angaben haben die Literatur lange Zeit belastet. Die Fäden bilden niemals "ein schönes anastomosierendes Netz" (Nadson, 1900, p. 36). Die auch schon von Bornet \& Flahault beobachteten keulenförmigen Anschwellungen der Fäden deutete er als "die Sporangien, in welchen sich viele kleine, rundliche, unbewegliche Sporen entwickeln. Die Sporen wachsen unmittelbar in ein Algenröhrchen aus" (p. 36). Ostreobium quekettii steht nach Nadsons Auffassung Phyllosiphon arisari sehr nahe; er gliedert sie bei den Phyllosiphonaceen ein und stellt diese Familie zu den Siphoneen.

Die Einordnung bei den Phyllosiphonaceen (Printz, 1926; Fritsch, 1935) wurde von einigen Autoren nicht übernommen, sie führen sie unter den Gattungen "incertae sedis" (Feldmann, 1954; Lund, 1959). Mit den Phyllosiphonaceen wanderte Ostreobium durch Klassen und Ordnungen; wir finden die Gattung bei den Siphonales (Parke, 1953), bei den Vaucheriales (Scagel, 1957), bei den Chlorococcales (Christensen, 1962; Parke \& Dixon, 1964, 1968) und schließlich wieder bei den Codiales (Parke \& Dixon, 1976). Die Zugehörigkeit von Ostreobium zu den Chlorophyta hatte Jeffrey (1968) durch pigmentchemische Untersuchungen an Material aus Korallen - Ostreobium reineckei Bornet gesichert. 


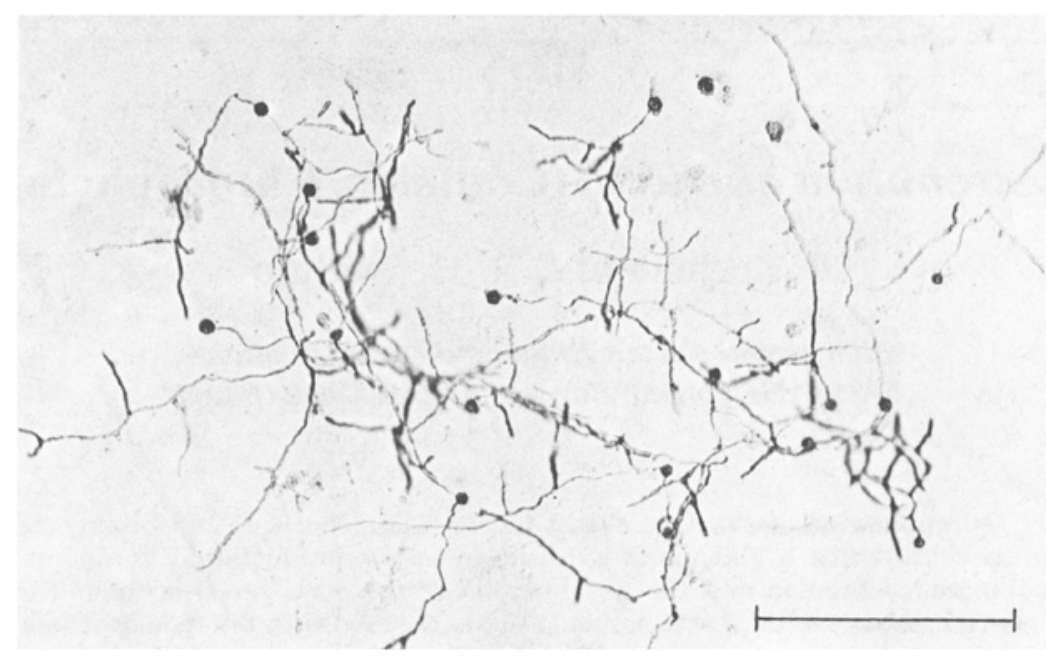

Abb. 1. Ostreobium quekettii, aus Schwärmern des Naturmaterials cntstanden. Näheres im Text. Maßstrecke $=300 \mu \mathrm{m}$

Anlaß zu einer nochmaligen Verwirrung gaben die Arbeiten von Mayhoub (1974a, b) über die Entwicklung von Pseudobryopsis myura. Aus der Zygote entsteht im Kulturversuch ein siphonales Fadenstadium, das morphologisch und zytologisch Ostreobium quekettii ähnlich ist. Bei Temperaturen über $19^{\circ} \mathrm{C}$ entsprossen diesem die aufrechten Thalli von Pseudobryopsis, einer Alge, die bei hoher Wassertemperatur und Lichtintensität im östlichen Mittelmeer optimale Bedingungen findet. Das kriechende, Ostreobium-ähnliche Stadium gedeiht während des ganzen Jahres hauptsächlich in größerer Wassertiefe "sur des coquilles mortes ou sur d'autres algues" (Mayhoub, 1974b, p. 167). An keiner Stelle findet sich eine Angabe, daß das fädige Stadium kalkbohrend ist, ein für Ostreobium quekettii wesentliches Merkmal. Wenn Parke \& Dixon (1976) Ostreobium quekettii als Sporophyten von Pseudobryopsis ansehen, so berechtigt Mayhoubs Darstellung nicht zu dieser Annahme. Wohl lassen die Autoren für Ostreobium quekettii aus britischen Gewässern die Möglichkeit offen, daß es sich hier um unspezifische Formen im Entwicklungszyklus von Bryopsidaceen handeln könnte. Auch diese Vermutung ist durch unsere Kulturversuche gegenstandslos geworden, die Ostreobium quekettii als selbständige Art auswcisen.

Dem ursprünglich von Muschelschalen als Substrat beschriebenen Ostreobium quekettii wurde später eine weltweite Verbreitung zugeschrieben. In jeder Art kalkhaltigen Substrats, auch in Korallen tropischer Meere, sollen diesc und vier weitere Arten der Gattung vorkommen. Eine zusammenfassende Darstellung gab Lukas (1974). Es wäre jedoch völlig zwecklos, am Schreibtisch mögliche Zusammenhänge oder Zugehörigkeiten dieser Formen erörtern zu wollen, von denen kaum mehr als der Name bekannt ist; auch bei ihnen ist eine Klärung nur durch Kulturversuche zu erwarten. An der Identität der bei Helgoland gesammelten Alge mit der typischen Art ist nicht zu zweifeln; daher sollte sich die Erforschung der Gattung an den hier mitgeteilten Ergebnissen ausrichten. 
Die ersten Beobachtungen für die vorliegende Studie wurden Ende August 1978 gemacht. In einer größeren Glasschale wurde eine von Tauchern besorgte Probe von Steinen und Muschelschalen aufbewahrt. An der Oberfläche des Wassers waren einige Deckgläser drei Tage lang exponiert, danach wurden sie in Nährlösung übertragen. Nach 22 Tagen waren die Deckgläser mit verzweigten Fäden bewachsen, die nur Ostreobium sein konnten (Abb. 1). Damit war erwiesen, daß Ostreobium in der Natur im Sommer bewegliche Fortpflanzungszellen bildet. Bis zur Klärung sollten aber noch fast zwei Jahre vergehen.

\section{MATERIAL UND METHODE}

Als Ausgangsmaterial diente kalkhaltiges Substrat aus etwa $6 \mathrm{~m}$ Tiefe, Muschelschalen oder Wohnröhren von Pomatoceros triqueter, deren grünliche Färbung die Besiedelung mit Ostreobium vermuten ließ. Aus dem zerkleinerten Material wuchsen in $\mathrm{GeO}_{2}$ haltiger Nährlösung neben Ostreobium auch fädige Grünalgen sowie Conchocelis und Cyanophyceen aus. Es gelang mit einiger Geduld, aus abgeschnittenen und in viel Flüssigkeit verteilten Fragmenten cyanophyceenfreie Ostreobium-Kulturen zu gewinnen. Die Alge gedeiht gut in Erdschreiberlösung bei $15^{\circ} \mathrm{C}$; in einer Nährlösung nach Provasoli mit einem Zusatz von Erdabkochung zeigte sich kein Unterschied. Die Kulturen erhielten 14 Stunden Licht von einer Tageslicht-Leuchtstoffröhre im Abstand von ca. $40 \mathrm{~cm}$.

\section{VERSUCHSERGEBNISSE}

\section{Sporangienbildung}

Unter den angegebenen Bedingungen regenerieren Ostreobium-Fäden in wenigen Wochen zu wattigen kugeligen Bällchen, die in älteren Kulturen 1 bis 1,5 cm Durchmesser erreichen. Die Fäden breiten sich auch rasch in Fragmenten von Muschelschalen aus. Durch gelegentliches Zerteilen der Bällchen und Übertragen kleinerer Flöckchen in frische Nährlösung lassen sich die Kulturen verjüngen. Über ein Jahr lang war nichts Auffälliges an ihnen zu beobachten, jedoch war auch nicht versucht worden, durch Änderung der Bedingungen eine Fruktifikation auszulösen; sie trat schließlich aus inneren Ursachen ein. Eine Woche nach dem Erneuern einer Kultur waren zahlreiche Fadenenden deutlich keulig angeschwollen und von dichtem dunkelgrünem Inhalt erfüllt. Abbildung $2(\mathrm{~A}, \mathrm{~B})$ zeigt ein schon etwas fortgeschrittenes Stadium mit unregelmäßig verzweigten, sackartigen Gebilden. Aus einigen ihrer blasigen Enden wächst ein kräftiger Faden mit hyaliner Spitze aus (Abb. 2 C); dieses Stadium war 14 Tage nach Versuchsbeginn erreicht. Ohne jeden Zweifel handelte es sich um junge Sporangien mit Entleerungstuben.

In frischer Nährlösung wurden die Sporangien nach weiteren 14 Tagen reif. Nur in wenigen Fällen blieben die Tuben so kurz wie in Abbildung $2 \mathrm{D}_{\text {; }}$ im allgemeinen waren sie sehr viel länger, und ihr Inhalt wandelte sich zu einer Schwärmerreihe um (Abb. 2 E). Ein solches Sporangium entleert sich gerade im mikroskopischen Präparat; seine unscharfen Partien lassen das Ausströmen der Schwärmer während der 5 sec dauernden Belichtungszeit erkennen. Es ist anzunehmen, daß die Sporangien vor ihrer 

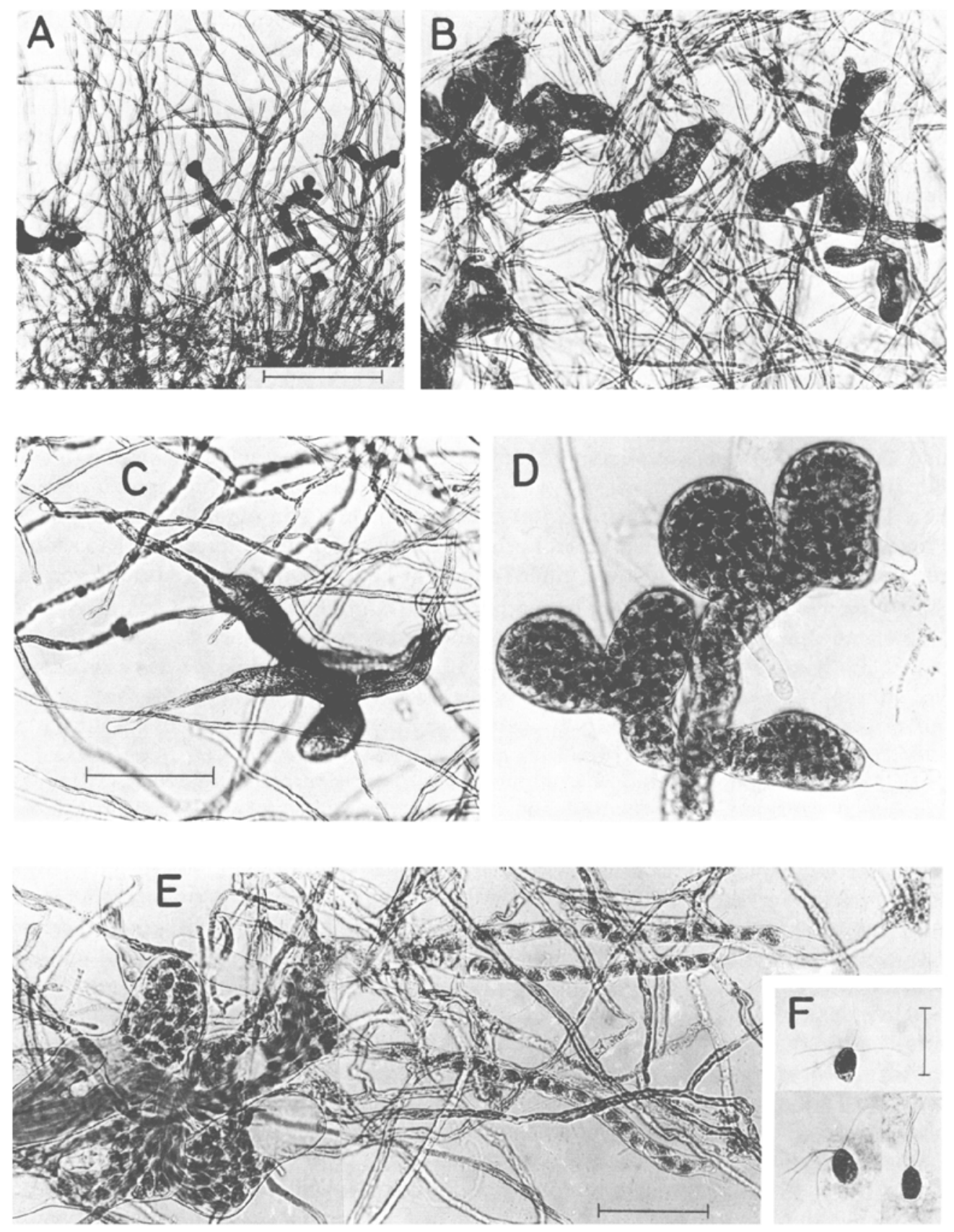

Abb. 2. Ostreobium quekettii. A, B Fäden mit Sporangienanlagen. C Junges Sporangium mit drei Entleerungstuben. D Reifes Sporangium mit kurzen Tuben. E Sporangium während des Ausschwärmens; Zoosporen gereiht in den langen Entleerungstuben. F Zoosporen. Maßstrecken: $A=200 \mu \mathrm{m}: \mathrm{B}, \mathrm{C}=100 \mu \mathrm{m} ; \mathrm{D}, \mathrm{E}=50 \mu \mathrm{m} ; \mathrm{F}=20 \mu \mathrm{m}$ 


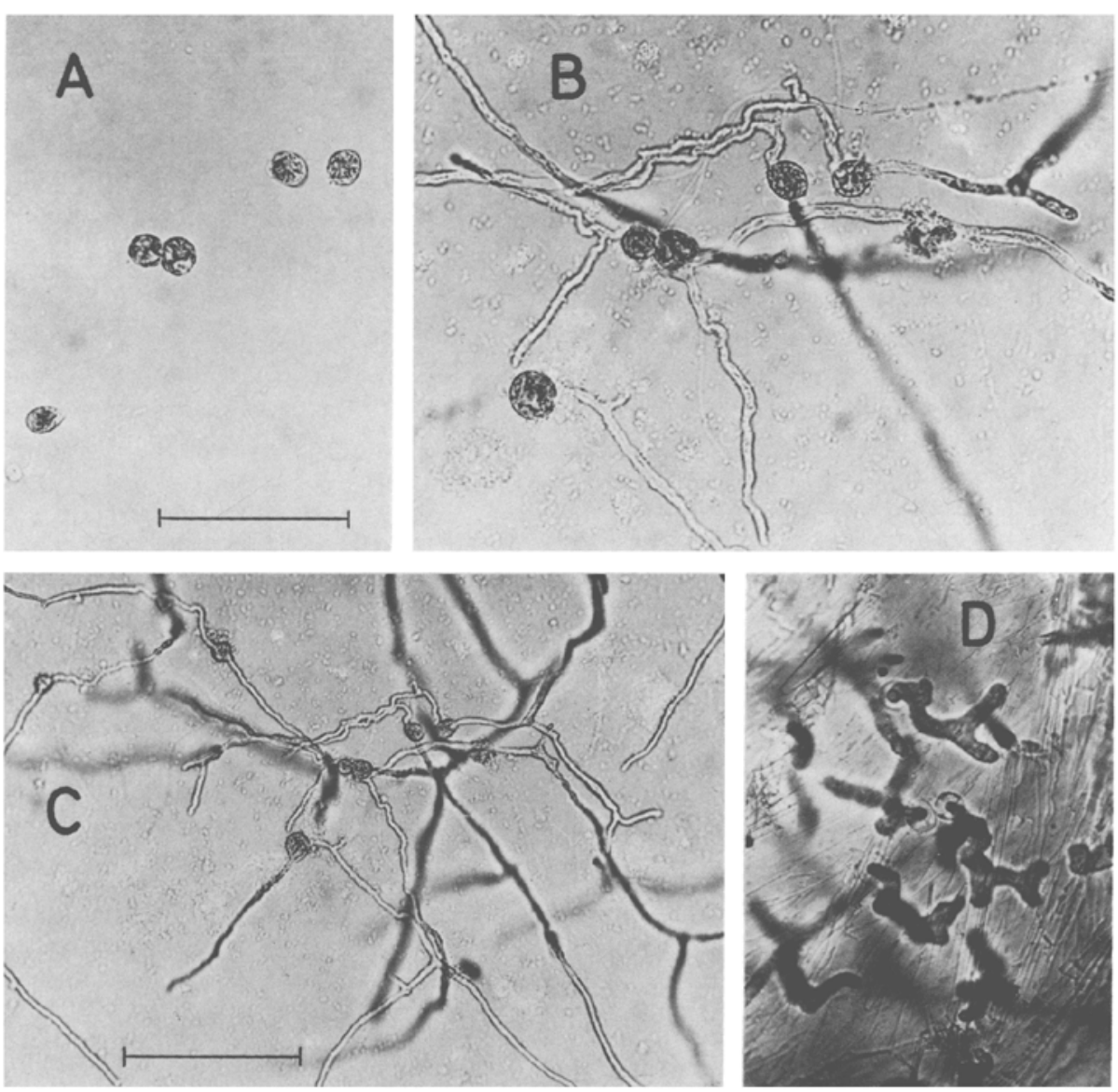

Abb. 3. Ostreobium quekettii. A-C Entwicklung einer Gruppe von 5 Zoosporen in freier Kultur nach 10 und 17 Tagen. D 5 Tage alte, in ein Muschelschalenfragment eingedrungene Keimlinge, die Sporenhülle ist auf der Oberfläche sichtbar, Maßsstrecken; $A, B, D=50 \mu \mathrm{m} ; \mathrm{C}=100 \mathrm{~mm}$

Reife durch einen Plasmapfropf von dem vegetativen Thallus abgetrennt werden; ein sicherer Nachweis gelang uns wegen der schwierigen Präparation des sehr empfindlichen Materials nicht.

Noch zweimal wurde die Stammkultur im Abstand von 14 Tagen emeuert: alle Regenerate in jeweils vier Plastikschalen wurden in der gleichen Weise fertil.

\section{Die Zoosporen und ihre Entwicklung}

Die ovalen, 9-12 $\mu \mathrm{m}$ großen Zoosporen tragen an ihrem spitzen Vorderende vier Geißeln von etwa doppelter Körperlänge (Abb. 2 F). Die beträchtlichen Größenunterschiede fallen an den beweglichen und an den zur Ruhe gekommenen Schwärmern auf. Ein schmaler Augenfleck ist vorhanden; die positiv phototaktischen Zoosporen setzen 

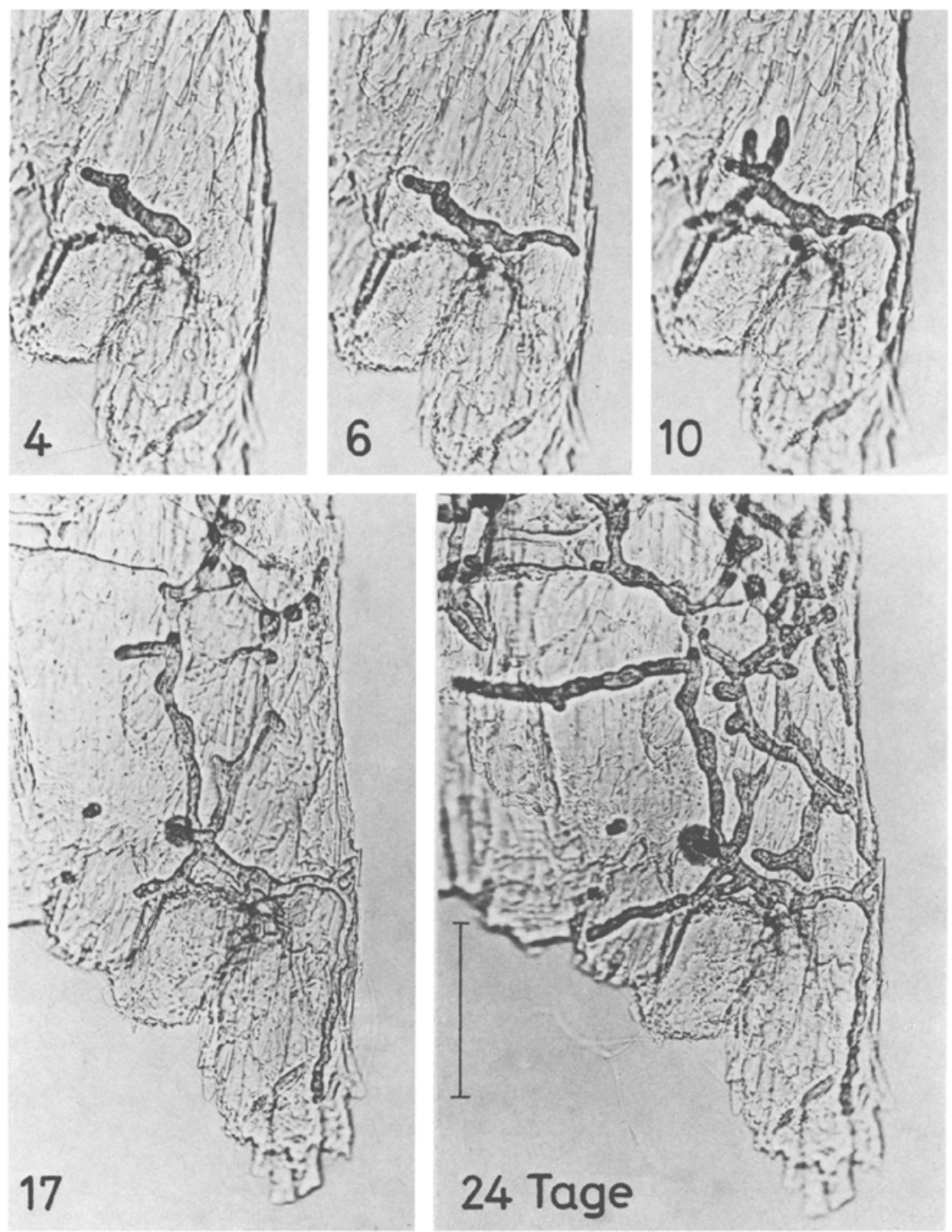

Abb. 4. Ostreobiun quekettii. Ausbreitung einer Pflanze in einem Muschelschalenfragment, die Zahlen geben das Alter der Kultur an. Maßstrecke $=50 \mu \mathrm{m}$

sich schnell fest. Sie entwickeln sich in freier Kultur und in Fragmenten von Austernschalen zu einer der Ursprungspflanze entsprechenden Generation.

In Abbildung $3(\mathrm{~A}-\mathrm{C})$ ist die Entwicklung einer Gruppe von 5 Zoosporen in freier Kultur dargestellt. Der unregelmäßig sich verzweigende Faden wird nicht durch eine Querwand von seiner Spore abgetrennt, sie behält stets ihre grüne Färbung, die sich sogar nach einigen Tagen wieder verstärkt. Im Alter von 19 Tagen (C) entsprach die Kultur der etwa gleichalten aus Schwärmern des Naturmaterials (Abb. 1). Bei diesem 

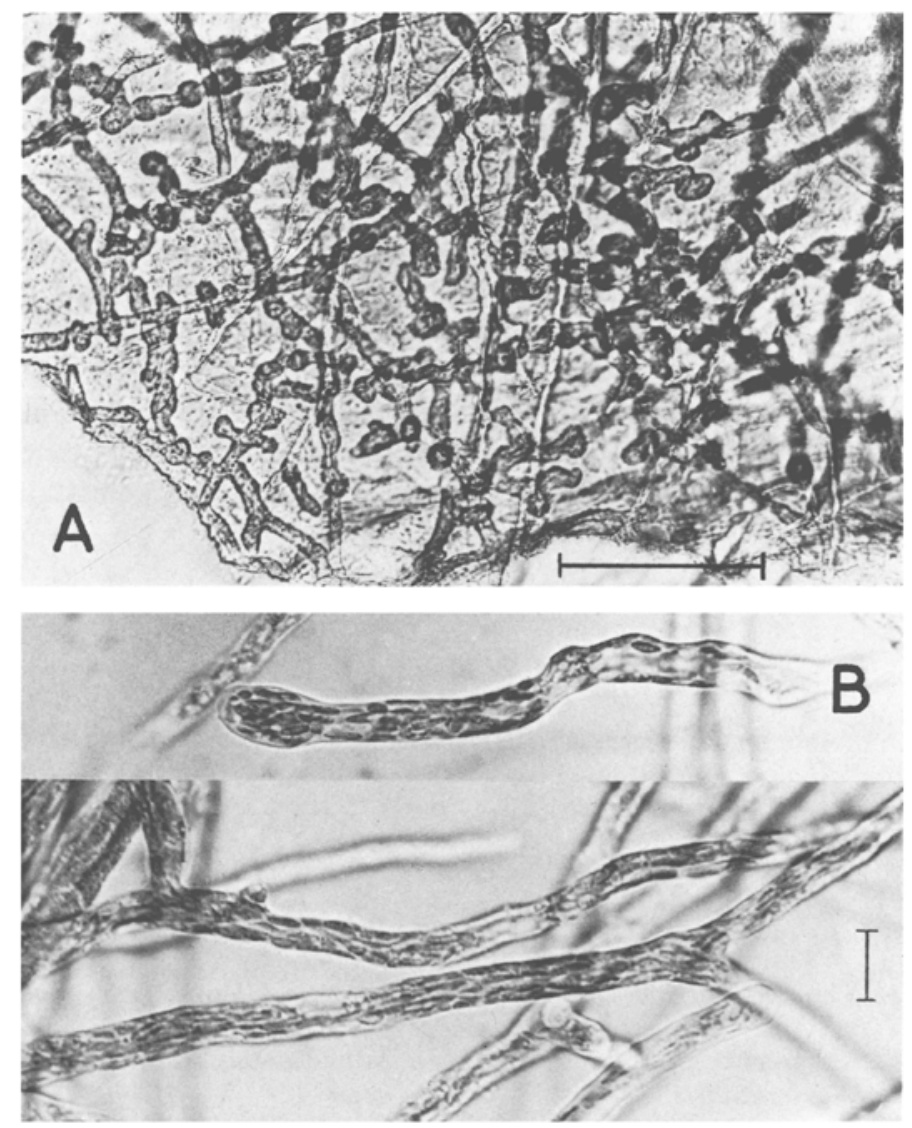

Abb. 5. Ostreobium quekettii. A Alterer Thallus in einem Muschelschalenfragment. B Freie Fäden mit Chromatophoren. Maßstrecken: $\mathrm{A}=50 \mu \mathrm{m} ; \mathrm{B}=10 \mu \mathrm{m}$

war der Anteil besonders dicker Sporenkugeln etwas höher als bei den kultivierten Pflanzen, so daß auch die meisten in der Natur entstandenen Zoosporen vielleicht etwas größer sein könnten.

Die auf einem Austernschalenfragment zur Ruhe gekommenen Schwärmer entleeren ihren Inhalt zunächst vollständig in den im Substrat sich unregelmäßig verzweigenden Keimfaden; die leere Sporenmembran ist auf der Oberfläche einer fünf Tage alten Kultur deutlich zu erkennen (Abb. $3 \mathrm{D}$ ). Auch sie füllt sich bald wieder mit grünem Inhalt. Wie rasch sich das Fadengeflecht aus einer einzelnen Spore in der Kalkschale ausbreitet, ist in einer Bildserie dargestellt (Abb. 4). Die Fäden passen sich lieber der Form des Schalenfragments an, als daß sie das Substrat verlassen. Wenn sie in älteren Kulturen doch herauswachsen, so hüllen sie das Substrat bald in einen dichten Pelz dünner, vorzweigter Fäden ein. Die spindelförmigen Chromatophoren ohne Pyrenoid sind unterschiedlich lang; in den Fadenenden und den dunkelgrünen, nicht von dem Faden abgetrennten Zoosporenhüllen sind sie besonders kurz (Abb. 5 B). 


\section{SCHLUSSBEMERKUNG}

Abbildung 5 A zeigt den in einer dünnen Kalkschale wachsenden, lebend photographierten Thallus von Ostreobium. Er entspricht gut den von Bornet \& Flahault (1889, Taf. IX, 5 und 6) gezeichneten Aufsichtsbildern auf eine nicht entkalkte Muschelschale. Die morphologische Übereinstimmung mit dem Typus erscheint daher hinreichend gesichert; auch sind die ökologischen Bedingungen an den von Bornet \& Flahault (1889) angegebenen Fundorten - Le Croisic, Brest, Normandie - mit denen Helgolands vergleichbar. Übrigens stammte auch das von Nadson (1900) untersuchte Material von Helgoland.

Als Ziel weiterer Kulturversuche wäre die Fertilisierung des in seinem Substrat wachsenden Thallus anzustreben. Zur vollständigen Klärung des Entwicklungszyklus sollte auch noch die Vermehrungsweise der aus den Zoosporen entstandenen Generation bekannt sein.

\section{ZITIERTE LITERATUR}

Batters, E. A. L., 1892. On Conchocelis, a new genus of perforating algae. In: Phycological memoirs. Ed. by George Murray. London, 1, 25-28.

Bornet, E. \& Flahault, Ch., 1889. Sur quelques plantes vivant dans le test calcaire des mollusques. Bull. Soc. bot. Fr. 36, 147-176.

Christensen, T., 1962. Alger. In: Botanik, Bd 2, 2. Ed. by T. W. Böcher, M. Lange \& T. Sørensen, Munksgaard, København, $178 \mathrm{pp}$.

Feldmann, J., 1954. Inventaire de la flore marine de Roscoff. Algues, Champignons, Lichens et Spermatophytes. - Trav. Stn biol. Roscoff. Suppl, 6, 1-152.

Fritsch, F. E., 1935. The structure and reproduction of the algae. I. Univ. Cambridge Press, London, $791 \mathrm{pp}$.

Jeffrey, S. E., 1968. Pigment composition of siphonales algae in the brain coral Favia. - Biol. Bull. mar. biol. Lab., Woods Hole 135, 141-148.

Lukas, K. J., 1974. Two species of the Chlorophyte genus Ostreobium from skeletons of Atlantic and Caribbean reef corals. - J. Phycol. 10, 331-335.

Lund, S., 1959. The marine algae of East Greenland. I. Taxonomical part. - Meddr Grønland 156 (1), $1-247$.

Mayhoub, H., 1974a. Reproduction sexuée et cycle du développement de Pseudobryopsis myura (Ag.) Berthold (Chlorophycée, Codiale). - C. r. hebd. Séanc. Acad. Sci., Paris (D), 278, 867-870.

Mayhoub, H., 1974b. Observations et explications au sujet de l'écologie et de la répartition du Pseudobryopsis myura (Ag.) Berthold (Codiales, Bryopsidacées). - Bull. Soc. phycol. Fr. 19, 164-167.

Nadson, G., 1900. Die perforierenden (kalkbohrenden) Algen und ihre Bedeutung in der Natur. Bot. Zap. 18, 35-40.

Parke, M., 1953. A preliminary check-list of British marine algae. - J. mar. biol. Ass. U. K. 32, $497-520$.

Parke, M. \& Dixon, P. S., 1964. A revised check-list of British marine algae. - J. mar. biol. Ass. U. K. 44, 499-542.

Parke, M. \& Dixon, P. S., 1968. Check-list of British marine algae - second revision. - J. mar. biol. Ass. U. K. 48, 783-832.

Parke, M. \& Dixon, P. S., 1976. Check-list of British marine algae - third revision. - J. mar. biol. Ass. U. K. 56, 527-594.

Printz, H., 1926. Die Algenvegetation des Trondhjemsfjordes. - Skr. norske VidenskAkad., 1926, (5), 1-274.

Scagel, R. F., 1957. An annotated list of the marine algae of British Columbia and Northern Washington. - Bull. natn Mus. Can. (Biol. Ser.) 52 (150), 1-289. 